\title{
A Herd Effect Detection Method Based on Text Fea- tures
}

\author{
Tingzhen Liu*, Tong Zhou, Yuxin Shi, Siyuan Liu and Jin Gao
}

College of Information Science and Engineering, Shenyang University of Technology, Shenyang 110020 China

* Correspondence: author: OCRID Email: firstsg@outlook.com

\begin{abstract}
The herd effect is a common phenomenon in social society. The detection of this phenomenon is of great significance in many tasks based on social network analysis such as recommendation. However, the research on social network and natural language processing seldom focuses on this issue. In this paper, we propose an unsupervised data mining method to detect herding in social networks. Taking shopping review as an example, our algorithm can identify other reviews which are affected by some previous reviews and detect a herd effect chain. From the overall perspective, the cross effects of all views form the herd effect graph. This algorithm can be widely used in various social network analysis methods through graph structure, which provides new useful features for many tasks.
\end{abstract}

Keywords: Social Networks; Data Mining; Graph Structure; Natural Language Processing; Machine Learning

\section{Introduction}

Herd behavior refers to the psychological process in which people make judgments and form impressions consciously or unconsciously according to the choices of the majority of people. The detection of herd effect is a problem worthy of study. The most concerned about herd effect is the field of behavioral finance. Because transaction data can be used for analysis, behavioral finance scholars have made a full research on it. Most detection methods are based on CSAD [1]. For example, ARCH [2] tests the non-linear relationship between the absolute deviation of cross-section returns and market returns to determine whether herding is significant. The CCK [3] model uses the cross-sectional absolute deviation between individual stock return and market average return and CAPM to judge the aggregation degree of investors. Some other works [4] use the dispersion of some index combinations to judge herd effect. In the field of recommendation algorithm, Wang et.al [5] proposed a recommendation algorithm that not only needs to consider personal information needs, but also needs to consider the influence of others in the society. That is, considering the influence of herding. Yang et al. [6] designed a model with herding effect attention mechanism to recommend videos to users through collaborative filtering.

In recent years, the application of graph based social network analysis[10] in practical scenarios is also a hot topic. Feng et al. [11] Analyzed the communication network of pyramid scheme organization. Gao [12] used link prediction for social network community mining. Huang et al. [13] proposed a high order structure called terminated harmonic motif for community detection. Wang et al.[14] uses the graph neural network to analyze the social network diagram 
and the relationship between users and products, and get the recommendation result. Wen et al. [15] used popularity in the community to restore the directionality of undirected social networks. Many studies on on opinion diffusion in social networks consider the herd effect. Tan et al. [7] studied the multi-source information diffusion in the presence of herding in online social networks. Zhanga et al. [8] Social temperature is proposed as an external factor to participate in the opinion evolution of nodes, which make the evolution of public opinion more accurate in the crowd. Das [9] studied the opinion formation process of social networks considering trust relationship. However, this kind of social network graph data often only comes from the user relationship graph of social software, which is often accompanied by the drawbacks of less relationships and inaccurate relationships.

In our work, natural language processing is used to analyze the herd effect in social networks. This work is still blank in the field of social network analysis. Take shopping reviews as an example. Reviews written by a user may be influenced by the reviews he or she has seen. This will have a chain effect. As shown in Figure 1 :

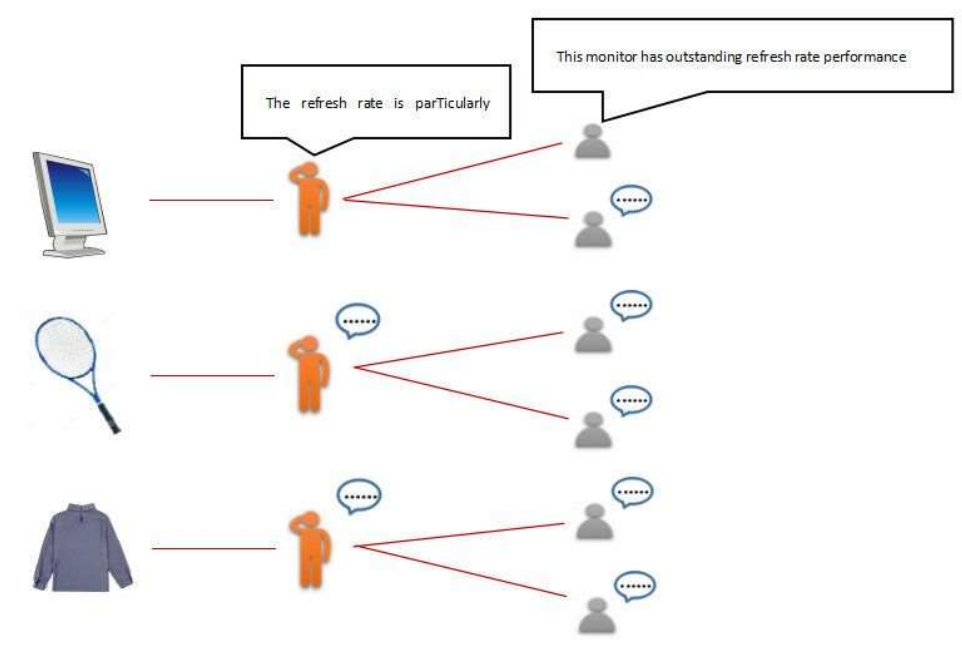

Figure 1. Herd effect in shopping Reviews.

Detecting the herd effect in social networks is of great significance in many analysis tasks. By understanding how the views of users in a community influence each other, we will have more evidence to judge the relationship among users in the community. This can enhance the accuracy of multiple tasks such as recommendation.

\section{Method}

\subsection{Metrics selection}

Our basic hypothesis is: If a user's review uses similar keywords to express the same meaning as a previous review (similar language patterns)[16]. We believe that he was influenced by previous reviews, that is, by herd effect. So we need to choose a measure to evaluate the similarity of the two review language patterns.

People usually use doc2vec[17] to get the vector describing the text, and then calculate the distance between the corresponding vectors of two texts to measure the semantic similarity. However, it does not apply to the herd effect detection task. Because if a product has an 
obvious advantage or defect, the user will have a high probability to review on this feature. These reviews express similar semantics, this will lead to similar doc2vec embedding. That is to say, if the herding detection model based on semantic judgment is used to judge two reviews that describe the same feature, then the later reviews will be judged to be affected by the previous reviews. But this kind of review which describes the same advantages or disadvantages of the product is not necessarily influenced by the predecessors[18].

In this case, we think it can be used as an indicator to measure the herd effect that uses TF-IDF [19] to measure the overlapping degree of keywords. This is because of the nature of TF-IDF: common high-frequency words can appear in any review, so the weight should be smaller. However, if a word with a lower frequency appears again, it is more likely to be generated by the influence of previous reviews, and it should have a greater weight $[20,21]$. That is to say, if many people describe a feature of the product, the words that often appear when describing the feature will not appear as keywords in the review detection. That is to say, the reviews that describe the same features of the product is difficult to be judged as affected by the herd effect. This advantage makes the TF-IDF value based on keywords become our preferred metrics.

\subsection{Herd Effect metric based on TF-IDF}

Based on this judgment, we use TF-IDF as a basis for evaluating word weights. Weighting techniques commonly used in TF-IDF information retrieval.

$\mathrm{TF}$ is an abbreviation for the term frequency, and its calculation formula is:

$T F_{w}=N_{w} / N$

Where $N_{w}$ is the number of occurrences of the term $w$ in a text. $N$ is the total number of entries in the text.It reflects the normalized number of times a given word appears in the whole text. The purpose of normalization is to prevent it from biasing towards longer files.

IDF is an abbreviation for inverse document frequency, and its calculation formula is

$I D F_{w}=\log \left(Y / Y_{w}+1\right)$

Where $Y$ is the total number of documents in the corpus. $Y_{w}$ is the number of documents containing the term $w$. The denominator plus 1 is to avoid the occurrence of the term not appearing in any document, which causes the denominator to be 0 . It reflects how often a word appears in all text. If a word appears in a lot of text, its IDF value should be low.

TF-IDF is a multiplication of the two. It integrates two indicators, TF and IDF, to evaluate the importance of a word to the entire corpus. The importance of a word increases proportionally with the number of times it appears in the file, and decreases inversely with 
the frequency of its appearance in the corpus. That is, the more times a word appears in a review, and the fewer times it appears in all documents, the more it can represent the review and distinguish it from other reviews.

\subsection{Constructing the graph of herding effect}

We use a link structure to describe the herd effect. Each review is treated as a node. If a review is affected by a review published before it, an edge is connected between them. We call the review herd review that influenced by the herd effect. Through the connection between review nodes, we can get some chains. Each node in these chains is affected by the herd effect produced by its precursor node. We call this kind of chain, influence chain.

It is worth noting that a node does not necessarily have only one successor. That is to say, a review may affect the judgment of multiple users and make the herd effect branch. Finally, we can observe the graph structure formed by many chains crossing. We call it influence graph. In this graph structure, we can observe the whole picture of the herd effect in a certain kind of product reviews. That is, how do different views influence each other.

After knowing the target, we consider establishing the connection between the review nodes according to the TF-IDF value. For ease of calculation, some words with lower TF-IDF values will not be considered. These words include symbols, stop words, personal pronouns and junctions. Words that appear only once should also be deleted. They may be numbers that are not helpful for analysis. After these processing, we can get a sparse TF-IDF matrix, which contains all the available keywords and their corresponding weights.

Then we iterate through these reviews in chronological order, letting subsequent reviews try to connect with some of the previous ones. As shown in Algorithm 1:

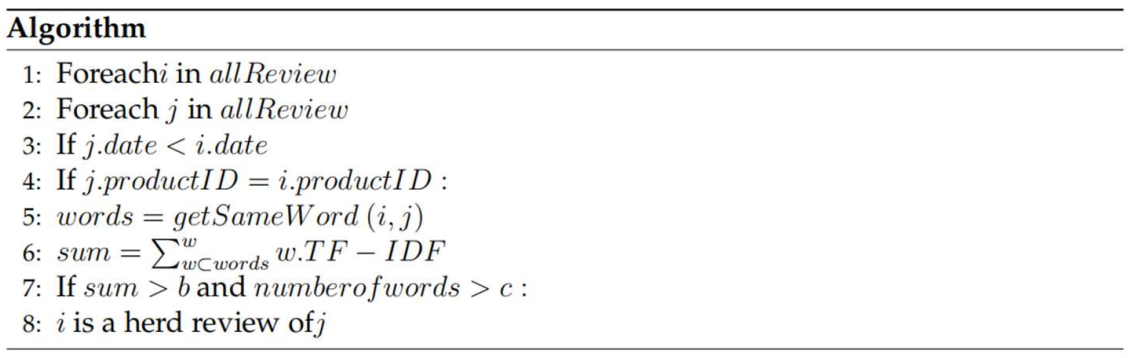

Alg.1.Establish edges between review nodes based on the TF-IDF Metrics.

It can be seen that if $i$ is a herd review of $j$, it must be satisfied that ${ }^{j}$ is published earlier than $i$ and both are reviews of the same product. On this basis, the two need to have enough common keywords (controlled by the hyperparameter c). The sum of the TF-IDF values of these keywords must also be greater than the threshold $b$.

If review $i$ is herd review of $j$, then the two will merge into a review chain. Reviews in the same Review chain are necessarily affected by a review in the chain that was published earlier than it.If a new review is a herd review of any review in a review chain, it is also merged into this chain. In this way, a traced influence chain is obtained. And then the influence graph is obtained. 


\section{Experiment}

Based on Amazon shopping review dataset, we analyzed the data of microwave oven, baby pacifier wipes and hair dryer, and established the herding model. The influence chain and influence graph were drawn by using the model created by Baby Pacifier's review data. We used Python to process the data in the three data sets. In terms of parameter selection, many reviews affected by herding can not be detected due to too large parameters, while many comments not affected by herding will be detected if the parameters are too small. After several experiments, we choose $b=3, c=3$ as the parameters. We get the following results:

\section{baby pacifier wipes}

Its influence chain diagram is shown in Figure 2:

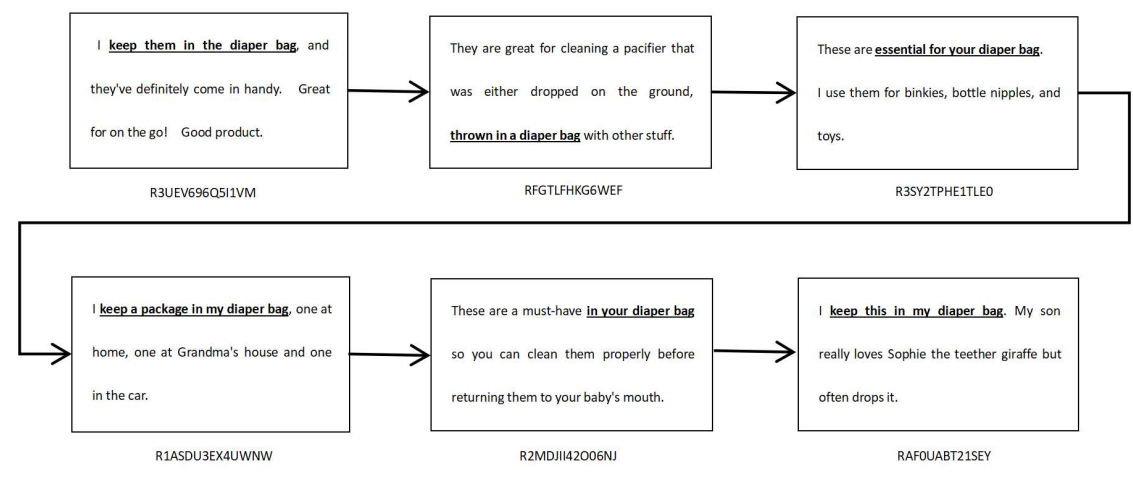

Figure 2. Influence chain of baby pacifier wipes.

This is part of the influence chain of a review on baby pacifier wipes. As we can see, the reviews in this part of the influence chain have "diaper bag" as a common keyword. This is probably because the first reviewers on the influence chain found that the baby pacifier wipes were suitable for putting in the diaper bag, which made successor node find this point after seeing these reviews and make similar reviews. Such logic is meaningful, which also reflects the accuracy of the model.

Its influence graph diagram is shown in Figure 3: 


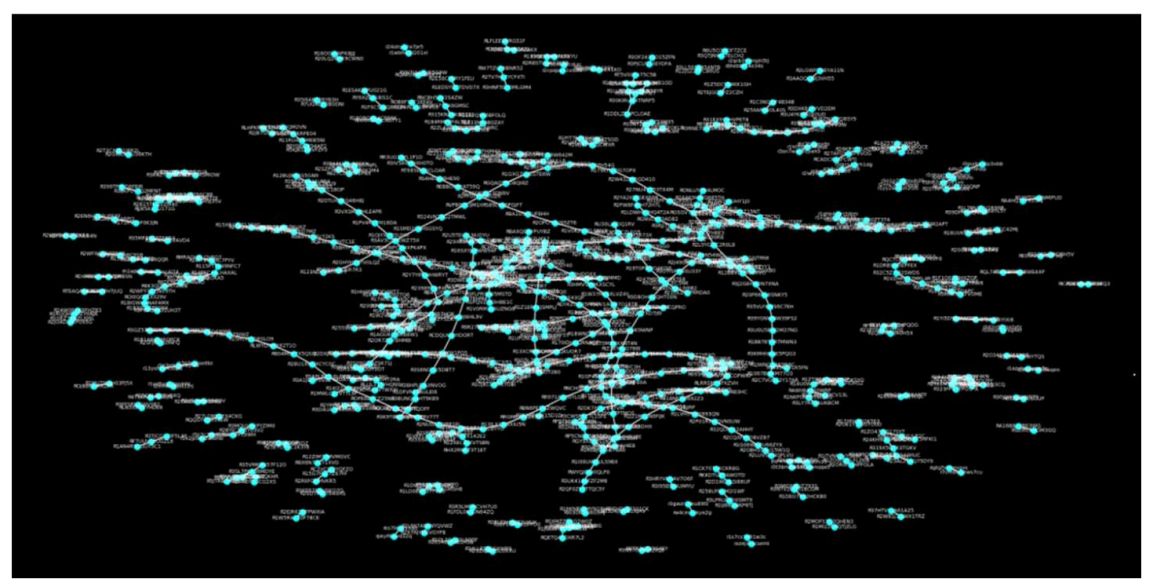

Figure 3. Influence graph of baby pacifier wipes.

The nodes on the graph represent each review of the product, and the label is the ID of the review in the dataset. We can see that the influence chain has a certain degree of overlapping, which means that some reviews have played an important role in multiple influence chains. Reaction on the product means that some people find some features of the product. These features are easily recognized by other users and lead them to write similar reviews. This leads to herd behavior. This shows that the product has many features that are easy to discover.

A total of 833 herd reviews are detected in 18,353 reviews of baby pacifier wipes. They make up 205 review chains. The size of each review chain is shown in Figure 4:

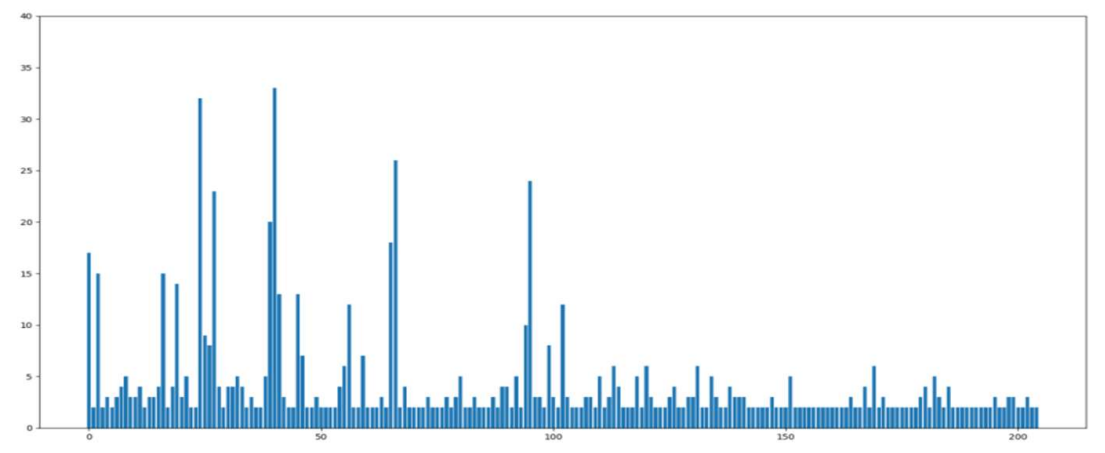

Figure 4. Influence chain length of baby pacifier wipes.

The maximum is 33 , the average is 4.06 , the mode is 2 , and the number of review that greater than average is 421 .

Similarly, a user has a $4.5 \%$ probability of making a herd review.There are 160 reviews generated by the herd effect, which means that a $2.3 \%$ probability of a user being affected by the herd effect makes herd review. Although the total number of baby pacifier wipes is many, the numerical characteristics are lower than those of microwave ovens that are ten times less.The evaluation of the baby's pacifier is very low due to the herd effect.

\section{Hair dryers}

A total of 1382 herd reviews is detected in 11,471 reviews of hair dryers. They make up 239 review chains. The size of each review 
chain is shown in the figure. Each bar represents a review chain, and the vertical axis is the number of reviews in Figure 5:

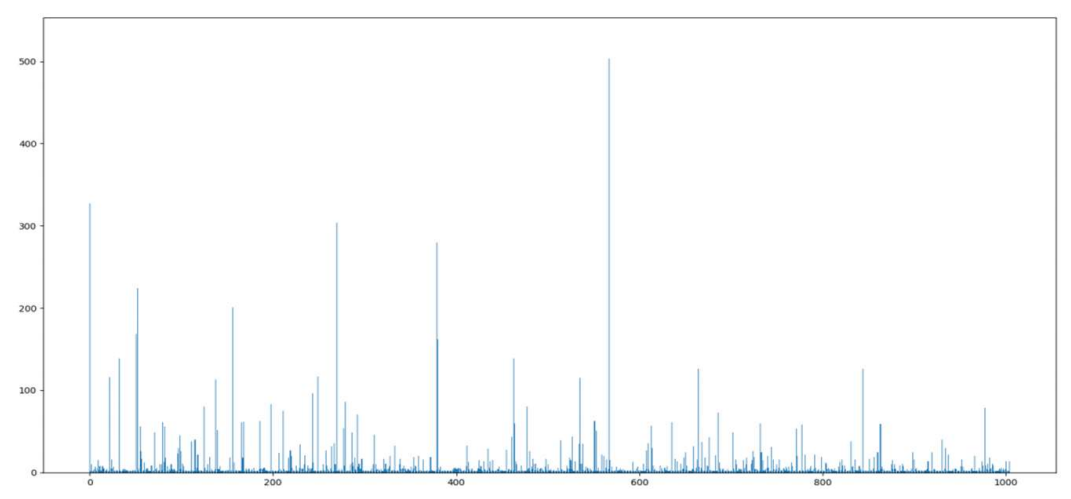

Figure 5. Influence chain length of hair dryers.

The maximum value is 81 , the average is 5.78 , the mode is 2 , and the number of review that greater than average is 903 .

We can see from the average that herd review is not very common. Fewer reviews had similar language patterns and were not enough to constitute a herd effect. Most review chains did not affect too many people and did not constitute a herd effect. We took the average as the boundary value and took a value of 6 . That was, when the number of reviews in the review chain was greater than 6 , it was considered to constitute a herd effect, and when it was less than 6, it was considered to have no herd effect.

From the data, there is 1,382 herd reviews among 11,471 reviews. In other words, a user had a $12 \%$ probability of making a herd review. Based on our definition of the chain size to achieve the herd effect, 903 reviews were generated by the herd effect. It showed that a user has a $7.8 \%$ probability to make a herd review by the herd effect.

\section{Microwave oven}

A total of 275 herd reviews were detected in 1557 reviews of the microwave oven. They make up 64 review chains. The size of each review chain is shown in Figure 6: 


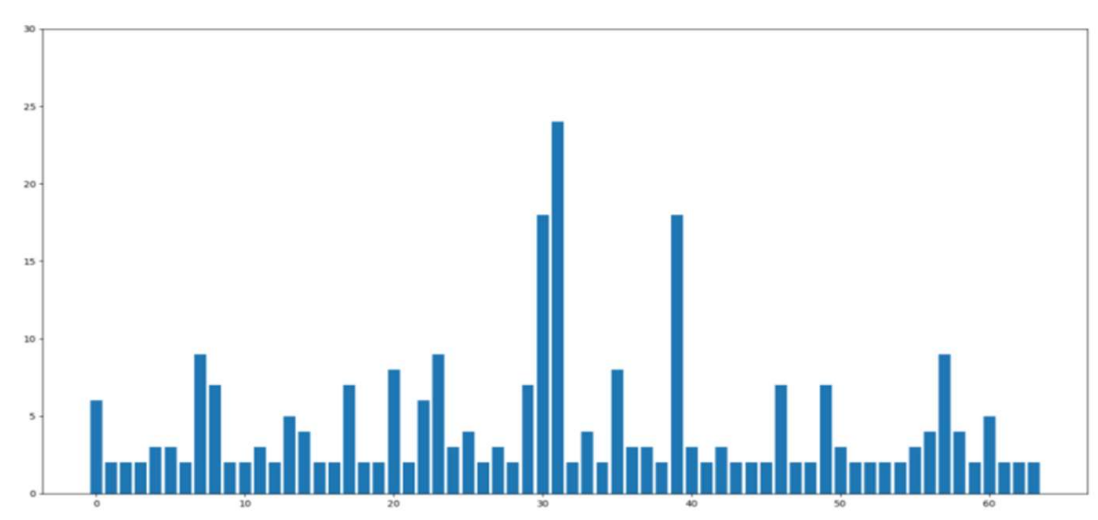

Figure 6. Influence chain length of Microwave oven.

The maximum value is 24 , the average is 4.29 , the mode is 2 , and the number of review that greater than average is 160 .

Similarly, there is a $17 \%$ probability that a user will make a herd review. There are 160 reviews generated by the herd effect, which means that a user has a $10 \%$ probability of making a herd review by the herd effect.

\section{Conclusion}

In this paper, we proposed a text herding detection model which filled in the blank of current research on this issue. It finded the influence chain of herding according to the language pattern of the text. The intersection of these influence chains further produced a directed influence graph.

This influence graph can provide a new basis for a variety of social network analysis tasks. For example, users in an influence chain may be more similar, and such judgments can be used as features of the recommendation system, so that the model can use more information to achieve higher accuracy.

\section{5 . Acknowledgments}

Thanks are due to Yizhong Xin for assistance with the experiments and valueable discussion.

\section{References}

1. Dai S G, Lu B. The Empirical Analysis of Herd Behavior in Stock Market Based on the CSAD Model[J]. Research on the Generalized Virtual Economy, 2016.

2. Barde S . Direct comparison of agent-based models of herding in financial markets[J]. Journal of Economic Dynamics and Control, 2016, 73(DEC.):329-353.

3. Yu Z, Zheng X. A Study of Herd Behavior Based on the Chinese Stock Market[J]. 2016.

4. Hudson Y, Yan M, Zhang D. Herd Behaviour \& Investor Sentiment: Evidence from UK Mutual Funds[J]. Social Science Electronic Publishing, 2020.

5. Wang, JyunCheng, Lin, J P. Are Personalization Systems Really Personal? - Effects of Conformity in Reducing Information[C]// Hawaii International Conference on System Sciences. IEEE, 2003.

6. Yang W, Gao W, X Zhou, et al. Herding Effect based Attention for Personalized Time-Sync Video Recommendation[J]. IEEE, 2019.

7. Tan Z, Wu D , Yang G, et al. APMSID: Activated Probability for Multi-Source Information Diffusion in Online Social Networks[J]. IEEE Access, 2018, PP:64435-64449.

8. Zhanga C, Lib C . Research on Spreading and Evolution of Opinion in Online Social Network[J]. Advanced Materials Research, 2013, 760-762:1982-1986.

9. RAJKUMAR DAS. Opinion Formation Modelling in Online Social Networks[D]. Monash University. 2018.

10. Xiao L, Wu X, Wang G. Social Network Analysis Based on Graph[C]// 2019 12th International Symposium on Computational Intelligence and Design (ISCID). 2019.

11. Feng P, Lu X, Gong Z , et al. Social Network Analysis Model for Research on Organizational Structure of the Pyramid Scheme Communication Network[J]. MethodsX, 2021, 8(81):101259. 
9

12. Gao Y . Constructing the social network prediction model based on data mining and link prediction analysis[J]. Library Hi Tech, 2019, ahead-of-print(ahead-of-print).

13. Huang L, Wang C D, Chao H Y . A Harmonic Motif Modularity Approach for Multi-layer Network Community Detection[C]// 2018 IEEE International Conference on Data Mining (ICDM). IEEE, 2018.

14. Wang X , He X , Chua T S . Learning and Reasoning on Graph for Recommendation[C]// WSDM '20: The Thirteenth ACM International Conference on Web Search and Data Mining. ACM, 2020.

15. Wen Y M , Huang L, Wang C D , et al. Direction Recovery in Undirected Social Networks Based on Community Structure and Popularity[J]. Information Sciences, 2018, 473:31-43.

16. Liu T, Chen K, Zhong Y . Threshold of Herd Effect for Online Events in China[J]. 2014.

17. Le Q V , Mikolov T . Distributed Representations of Sentences and Documents[J]. 2014.

18. Heng Y, Gao Z, Jiang Y, et al. Exploring Hidden Factors behind Online Food Shopping from Amazon Reviews: A Topic Mining Approach[J]. Journal of Retailing \& Consumer Services, 2018, 42:161-168.

19. Jing L P , Huang H K, Shi H B . Improved Feature Selection Approach TFIDF in Text Mining[M]. 2002.

20. Xingru A. The Research on the Threshold of High-Frequency Words Based on the Normal Distribution in Word Frequency Analysis[J]. Journal of Intelligence, 2014

21. Syafiandini A F , Mustika H F , Manik L P , et al. Implementing Graph Based Rank on Online News Media Keyword Extraction[C]// 2019 International Conference on Computer, Control, Informatics and its Applications (IC3INA). IEEE, 2020. 